MUSIC IN SHADE OF TECHNOLOGICAL DEVELOPMENT BETWEEN

\title{
HARM AND BENEFIT
}

\author{
Marwa Youssef ALSAYAD *
}

Theory and Composition Department, Faculty of Music Education, Helwan University, Egypt

\begin{abstract}
The twentieth century saw unprecedented technological development and openness, which began in the 19th century and reached its peak by the end of the 20th century. This development has taken on all aspects of life, including music. With globalization, the tremendous progress in communication and the availability of the Internet - which has become a living standard - the spread of this scientific and technical development has become very rapid. A long time ago, people started to look at music differently, and the modern medical attention went towards discovering the positive physiological and psychological effects of music. Music is no longer limited to be used as a treatment for grief or to express feelings or used in group therapy sessions, but it went far to the extent of the production of electronic devices to launch sound waves for holistic therapy and direct impact on the health of the individual. However, as much as the importance of this technical and scientific progress and its great potential for treatment is concerned, it is a two-edged sword. Some have started to exploit what scientists have reached in this field negatively, such as the use of narcotic music for the sake of financial profit. So sound therapy and music is a public benefit that has become a health hazard. This research deals with the positive therapeutic possibilities of music using modern technological means in addition to explaining the harmful music, which depends on the same technology and its impact on the human brain in order to warn people from it, along with emphasizing the need to deal with music with its useful logic and how to benefit from it.

Music Therapy, Digital Drugs Music, Binaural Beats, Brainwaves, Sound Waves.
\end{abstract}

\section{Introduction}

The twentieth century witnessed unprecedented technological development and scientific openness, the seeds of which began in the nineteenth century and reached its climax at the end of the twentieth century, and this development affected all areas of life, including music, of course. With globalization and the tremendous progress in the means of communication and the availability of the Internet - which has become one of the basics of living - the spread of that scientific and technical development has become very rapid.

Society's view of music differs from another societal category and differs according to its purpose. There are those who deal with music as a commodity and a commercial product, and there are those who have restricted their view of music to being an art of entertainment. However, a good number of scientists and doctors have found in music other dimensions that give it importance and depth, which makes it rise to the rank of science. In the late sixth century. The modern medical interest has appeared in discovering the positive physiological and psychological effects of music, and with the great technological development it is no longer confined to the use of music as a treatment for cases of sadness or to express and vent the limit

\footnotetext{
* Corresponding author: info@music.helwan.edu.eg
} 
of producing electronic devices to trigger waves of emotions or to use them in group therapy sessions, but beyond that to sound therapy Total, topical and direct impact on health.

\section{Research problem:}

Scientists and doctors have tended to use modern technological means to exploit music and sound frequencies in the treatment of many organic and psychological diseases because of their great effects on the brain and the nervous system, which is what we must go to study and conduct more research on it in order to benefit from it. Despite the importance of this technical and scientific progress and the great capabilities it made available in the field of sound therapy, it is a double-edged sword, as some have tended to exploit what scientists have reached in this field, exploiting a negative that is harmful to the health of the individual, after that the aim of sound and music therapy was to benefit The public has become a health hazard.

\section{Research terms:}

Sound therapy is the use of sounds and frequencies that have a specific effect on the tissues and organs of the human body with the aim of changing them to bring the individual to a healthy state. Music Therapy Music therapy is the exploitation of music with its various elements (rhythm - melody - harmony) through a therapeutic relationship established between the patient and the therapist who supports and improves the physical, mental, social or emotional state of the individual.

\section{Frequency}

The velocity of vibration of the bodies, measured by the number of compressions and vacations produced by a vibrating body per second.

\section{Binaural beats.}

It is the effect produced by listening to two sound waves with different frequency, and it is calculated as the difference between the two frequencies.

\section{Entrainment:}

It is one of the concepts of the psychology of sound, and here it means the process of changing brain waves, the speed of breathing, feelings, or thoughts by synchronizing with an external stimulus (rhythmic or musical).

\section{State of mind:}

The researcher believes that the concept of mental state in the current research expresses the amount of human brain activity and the degree of consciousness, which ranges from deep sleep, relaxation, alertness, and focus. It is associated with delta, alpha, and beta brain waves.

\section{The healing powers of music:}

Research dealing with music therapy has witnessed great interest in the twentieth century, as 
many studies highlight the importance of music in human life. Researchers have tended to prove the therapeutic capabilities inherent in music, sound frequencies and rhythms and how to benefit from them in a scientifically intended manner.

One of those studies was a study entitled "Difference in structure The brain between musicians and non-musicians "Differ between Musicians and Non - Musicians Brain Structures" by Christian Gaser and Gottfried Shlaug, where they studied the apparent differences between the brains of musicians and others who do not study music and amateur musicians, and they found that music students learn fine motor skills. And complex auditory at a young age (for example, the performer translates the musical notes he read into kinesthetic commands while observing the same moment auditory), and using measurement techniques they found differences in the area of gray matter that contains most of the larger bodies of non-musicians and amateur musicians.

Although some of these differences may be attributed to the individual's country readiness, the researchers are certain that they may be the result of the adaptation of the brain structure in response to the long-term acquisition of these skills and repeated training on them, which can be proven by more research in this area. Another study also showed that musicians actually have better memories than non-musicians.

Also among the most famous research in the field of musical sound therapy were studies by the researcher Royal Rife in 1888-1971, an American bacteriologist, to find a cure for cancer. He came to invent a microscope device that enables it to determine the frequency of chemical components of cancer cells, and to destroy them by using the same frequency for each virus by means of electronic frequencies of Frequency synthesis devices.

Research continued on the results of Rife's findings for treating cancer, as Fabien Maman and Helene Grimal studied the effect of music and singing on human cells in the seventies, and these experiments imaged the internal structure of each cancer cell as a photograph to follow the cell's reaction to The sounds you are exposed to. It was found that with the progressive ascending the music ladder, cancer cells began to explode at a certain frequency. Experiments recorded the best results with the use of the human voice in singing the musical scale.

As for Dr. Alfred Tomatis - 1920-2001, ear specialist and frequency researcher and one of the most important pioneers Music and therapeutic sounds, as he developed a method for retraining the middle ear muscles by means of extensive auditory techniques, and by simple sounds that could treat many conditions such as learning disabilities, autism, depression, chronic fatigue, or immune system disorders, and he was also able to help learn foreign languages in a way. Hurry up, communicate better with others, or improve creativity and business performance. 
Tomatis used the electronic ear (EE), a device he developed himself, through which he displays Mozart's music, ancient Gregory chanting, human voices, and special frequencies, and Mozart's music, which he used, proved its effectiveness without other than others, especially the Violina Concerto.

All these applications that were conducted and others confirm the effect of the sounds and vibrations around us on our health. Most conscious people know the impact of music on their psychological state and use the music they deem appropriate at home automatically to improve their mood or calm anxiety that may affect them or as an aid to sleep in cases of insomnia. However, researchers and scientists have conducted many researches in this field and have already come up with a clear formula and specific specifications for the appropriate music for each disease or imbalance that occurs in the individual's health, and through modern technology, computers and the Internet, each of these diseases has become very effective and available music treatments for everyone.

One of the most important recent applications that researchers have reached in the field of music, sound frequency and rhythm therapy using modern technology is the so-called brain wave entrainment.

\section{Brain wave entry:}

All of the human body's muscle, nervous, respiratory, and blood systems all operate according to a specific rhythm. The heart rate and pulse rate have a constant rate that is a measure of an individual's health. And blood flows in the body in rhythmic pulses based on the heartbeat, just as breathing must be calm with a regular rhythm, or from the brain, the speed of its brain waves changes in one day according to the state of consciousness and activity in which the individual is, knowing that each state has Steady rhythm.

The effect of rhythm on the body can be observed in two directions, internal to internal, where all the pulse systems in the human body coincide naturally with each other, for example, the speed of the heartbeat coincides with the speed of breathing, so if the heart rate increases, the speed of breathing increases automatically. And an external direction (External to Internal) where the voice is used as an influence to make the change happen

On one of the body parts, the speed of the brain waves can coincide with the music, and when the pulse of one of the organs changes with music, such as the brain, we find that the rest of the main pulse systems in the body, such as the heart and breathing, change accordingly.

The process of attracting brain waves is based on changing the speed of the individual's brain waves in order to treat some disorders such as insomnia or lethargy and other disorders that will be discussed in detail later. The attraction process depends on a special type of music that 
has the ability to make that change, and to know how to use this technology in treatment, the concepts of Resonance and Entrainment must first be addressed, as they are the basis of the science of sound therapy with all its techniques and applications.

Resonance is the most fundamental physical principle in using sound and rhythm as a therapeutic method. Each body in the universe has its own natural vibrational rate, and it is necessary to know the natural resonance of bodies when trying to influence those bodies or adjust their intensity.

As for the attraction, it is the process by which the natural frequency of the bodies is changed, that is, for a vibrating body to affect another body and change its vibrations, and for the attraction process to occur, it is required that the second body has the ability to reach the same required rate of oscillation.

Knowing the concepts of resonance and attraction helps us to know the way in which external tones and rhythms can bring about healing and create a positive effect on the body. Based on these two concepts, the researchers realized the possibility of changing the individual's brain wave frequencies in proportion to his pathology.

During the day, the individual experiences different mental states such as sleep, focus, work, creativity, meditation and other states, and each mental state has its own electrical signal that appears in the brain known as brainwaves. The brain consists of billions of neurons that communicate with each other using electricity, so these are billions of neurons. It generates a large capacity of electrical activity and with the repetition of that activity, repeated patterns of brainwaves arise and are measured with an EEG. EEG (Electroencephalogram) brain wave patterns are classified according to their frequency, which is measured by the number of vibrations per minute $(\mathrm{Hz})$. Often mental states associated with the patterns of brain waves oscillate between four specific mental states, namely:

\section{Psychedelic music:}

Also known as Digital Drugs "or" iDoser ", and according to the idoser website, which is the most famous in this field, it is an MP3 audio file of selected music or sounds with the addition of binaural frequencies to it, and in the same manner it is heard via headphones in both ears, so that Broadcast a specific frequency in the right ear and a different frequency in the left ear.

After researching a number of websites related to narcotic music, the researcher found that the promoters of that type of drug say that by studying the brain and the nature of the electrical signals that emanate from the brain after using a specific type of drug, it is possible to determine the desired state of ecstasy, as each type of drug Digitalis can target a specific pattern of brain activity. For example, when you hear the cocaine frequencies for minutes counted, this will 
prompt the brain to be stimulated in a manner similar to the image that it is stimulated in after realist abuse of this drug.

The damages of this narcotic music are severe and destructive, as it may aim to suppress or disable the functions of the nervous system and brain, and thus it leads to failure in breathing functions, loss of consciousness, and narrowing of the pupil. Or it may stimulate the nervous system and brain and thus lead to an increase in the respiratory rate, blood pressure, rapid heart palpitations and convulsions, and dilated pupils as they respond to light.

There are several sites on the Internet that offer and market digital drugs, and even provide several types of them that differ according to their effect on the individual. A written guide is also attached to it explaining the procedures that the listener must take in order to achieve the desired effectiveness. Every audio recording has its own potion and some of it has a specific sequence in listening to it.

According to what is mentioned on these sites, the doses they provide simulate the same effect as real drugs. Some sites did not stop at this only, but some of them offer the possibility of designing a special audio file in the event that an individual wants to access.

The particular feel is not from the recordings already within their library. Here are some examples of types of digital drugs offered by a website on the Internet: Absinthe, an intoxicating green drink that has been banned for a very long time. The website assumes that listening to the audio recording of this type - which has the same name - leads to the individual feeling that he is drunk, but at the same time he feels high mental concentration, so he speaks slowly and the words he says become very clear and detailed.

ACID, also known as LSD, is a hallucinogenic drug made from lysergic acid, which is a natural chemical found in a type of fungus. When listening to this audio recording for a period of 30 minutes, a general change in consciousness occurs, and the individual feels difficult to concentrate, with unusual symptoms such as redness of the face, chills, and a high body temperature), the individual experiences abnormal thoughts and speaks illogical words, and emotions change rapidly ( Happiness, fear, anxiety, anger, joy, and irritability). According to the makers of this record, this dose is very strong.

ADRENALINE doses for 20 minutes. It causes the blood vessels and airways to dilate so that the body can pump more blood to the muscles and get more oxygen in the lungs in a short time, which increases physical performance for short periods of time. This dose gives the individual a real experience of feeling the need to face danger or any situation that requires strong energy and self-confidence.

ADRENOCHROME, a disorientation and hallucinogenic sound recording, has such a 
powerful effect that the website it provides confirms that its use is at the individual's own risk. Anger, joy, and irritability). According to the makers of this record, this dose is very strong.

ADRENALINE doses for 20 minutes. It causes the blood vessels and airways to dilate so that the body can pump more blood to the muscles and get more oxygen in the lungs in a short time, which increases physical performance for short periods of time. This dose gives the individual a real experience of feeling the need to face danger or any situation that requires strong energy and self-confidence.

ADRENOCHROME, a disorientation and hallucinogenic sound recording, has such a powerful effect that the website it provides confirms that its use is at the individual's own risk. AlcoHOL wine has a 35-minute dose, and gives the same effect to different types of wine, such as beer and wine, so it works to feel relaxed with a sense of dizziness and imbalance, and leads to improved mood and a sense of happiness, and the individual does not find any contraindications to do anything he desires. The website markets this type of record as being as strong as drinking five glasses of gin wine.

Benz0 FURY mimics the effect of MDMA and amphetamine, so that when hearing it, the individual feels intense excitement and excitement, an increased energy in the body, with visual and auditory hallucinations. Physical side effects can include unpleasant effects such as an increased heart rate and a high body temperature, and they can also include unpleasant mental effects such as anxiety, panic, confusion, and weakness.

\section{Concentration.}

Some doctors have questioned the effectiveness of narcotic music, as they think that it does not affect the nervous system or the brain, but rather it is just a phenomenon common among young people and no more, and others believe that its effect is already present, but it is a temporary effect that disappears as soon as the vocal stimulus disappears, and does not reach The individual is subject to an addiction. But the researcher believes that the binaural technique has proven its effect on brain waves as previously explained, and therefore it may indeed be very effective in the case of narcotic music, as you see that even if its effect is temporary, as some doctors assert, this effect in the long term can harm Of the human brain and nervous system. In order to ensure the validity of these dangerous effects of this technology on the health of the individual, all attempts to spread these digital drugs in Egypt must be suppressed.

\section{Results:}

1- The binaural beating technique depends on the use of two different tones in their frequency, resulting in the difference between them a third frequency, which has the ability to affect the human brain and change his brain waves, thus changing his state of mind. 
2- A lot of research has been done on this technique and has proven its effectiveness in treating many mental disorders such as (fatigue, headaches and migraines - cognitive deficiency insomnia - organic pain - imbalance in hormone secretion - sleep problems and stress). It can also be used to improve capabilities. The individual's mentalities such as focus - creativity, memory - learning - meditation mood - intelligence level - mental skills).

3- Narcotic music is a phenomenon that has spread in the recent period and depends on the technique of beating the ears, but it aims to create abnormal mental states that are similar to the mental states that an individual experiences when consuming drugs or intoxicating substances, and for this it is necessary to emphasize the need to educate young people about their danger and distance from them, And inform the competent authorities to block the websites you promote.

4- Binaural frequency technology can be used with people suffering from ADHD, and the researcher also suggests researching the possibility of using this technique in psychotherapy, neurological and addiction treatment clinics.

5- The field of sound therapy is one of the important fields in our life, it depends on the use of musical elements such as frequencies and rhythms, and it has no side effects when using it to achieve the health of the individual such as drugs, and for this it is necessary to encourage scientific research in it, and send researchers to study in specialized institutions in This area outside Egypt to benefit from the results they have reached in our academic institutions.

\section{References:}

1- Bunt, Leslie. Music therapy, The New Grove Dictionary of Music and Musicians. (New York: Oxford University Press, 2001) vol.18

2- Binaural beats and Isochrones Tones 101, The Growthology Series, http://growthology.com 2011.

3- Campbell, Don and Alex Doman, Healing at the speed of sound. (New York: Plume, 2012).

4- Cruceanu, Victor D. and Violeta S. Rotarescu. Alpha Brainwave Entrainment as a Cognitive Performance Activator. Cognitiei, Creire, Comportment. Vol.17, No.3, September 2013.

5- Gaser, Christian and Gottfried Schlaug. "Brain Structures Differ between Musicians and Non-Musicians." The Journal of Neuroscience: The Official Journal of the Society for Neuroscience 23, no. 27 (October 8, 2003) .

6- Goldman, Jonathan. Healing Sounds: The Power of Harmonics. (Vermont: Healing Arts Press, 2002) .

7- Gray, Tim. The use of Sound for Control, Healing, and Empowerment. Unpublished master's thesis. (Mills College, 2005) 
8- Huang, Tina L. and Christine Charyton. A Comprehensive Review of the Psychological Effects of Brainwave Entrainment, ALTERNATIVE THERAPIES, Sep. /Oct. 2008, VOL. 14, NO. 5.

9- Huang, Zhen. Verbal memory retrieval engages visual cortex in Musicians. Neuroscience (June 16, 2010).

10- Jirakittayakorn, Nantawachara and Yodchanan Wongsawat, A Novel Insight of Effects of a 3-Hz Binaural Beat on Sleep Stages During Sleep. Front. Hum. Neurosci. 24 September 2018. 11- Lane, James D., Stefan J. Kasian, Justine E. Owens and Gail R. Marsh. Binaural Auditory Beats Affect Vigilance Performance and Mood. Physiology \& Behavior, Vol. 63, No. 2, , 1998. 12- Leeds, Joshua. The Power of Sound. (Vermont: Healing Arts Press, 2001).

13- Lind-Kyle, Patt. Heal Your Mind, Rewire your Brain (Santa Rose: Energy Psychology Press, 2009) .

14- Lynes, Barry. The Cancer Cure That Worked. (Lake Tahoe: BioMed Publishing.

15- Maman, Fabien. The Role of Music in the Twenty-first Century. (Tama-Do Press, 1997) . 16- McBride, Rhonda S. Peace, Power, and a Sound Mind: An Emerging Approach in the Treatment of Addictions. (Bloomington: Balboa Press, 2011) .

17- Oster, Gerald. "Auditory Beats in the Brain," Scientific American 229 (October 1973).

18- Rhoades, Rodney and David R. Bell, Medical Physiology: Principles for Clinical Medicine, (Baltimore: Lippincott Williams \& Wilkins, 2009)

19- Nevin Wagih KASSAB, CULTURAL AND CREATIVE INDUSTRIES IN THE ARAB REGION THE COMPLEMENTARITY OF THE RELATIONSHIP BETWEEN THE ECONOMIES OF CINEMATOGRAPHY AND THE EGYPTIAN NATIONAL INCOME, International Journal of Humanities and Language Research, Vol. 1, No. 1, 2018, pp. 24-29.

20- https://www.binauralbeatsmeditation.com/.

21- https://blog.bulletproof.com/binaural-beats-sleep-meditation-benefits/

22- https://drugabuse.com/lsd/effects-use/

23- http://growthology.com/binaural-beats

24- http://idosersoftware.com/index

25- https://insighttimer.com/meditation-topics/binauralbeats

26- https://www.tech-wd.com/wd/2014/11/15/4, Jall-digital-drugs-o/

27- http://www.tomatis.com

Received: August 22, 2018

Accepted: October 30, 2018 